\title{
Exploration of the Direct Use of Anodized Alumina as a Mold for Nanoimprint Lithography to Fabricate Magnetic Nanostructure over Large Area
}

\author{
M. Tofizur Rahman, Hao Wang, and Jian-Ping Wang \\ MINT Center, Department of Electrical and Computer Engineering, University of Minnesota, 200 Union Street SE, \\ Minneapolis, MN 55108, USA \\ Correspondence should be addressed to Jian-Ping Wang, jpwang@umn.edu
}

Received 4 August 2011; Accepted 24 August 2011

Academic Editor: Chuan Jian Zhong

Copyright $\odot 2011$ M. Tofizur Rahman et al. This is an open access article distributed under the Creative Commons Attribution License, which permits unrestricted use, distribution, and reproduction in any medium, provided the original work is properly cited.

We have explored the direct use of anodized alumina (AAO) fabricated on an Si wafer as a mold for the nanoimprint lithography (NIL). The AAO mold has been fabricated over more than $10 \mathrm{~cm}^{2}$ area with two different pore diameters of $163 \pm 24 \mathrm{~nm}$ and $73 \pm 7 \mathrm{~nm}$. One of the key challenges of the lack of bonding between the antisticking self-assembled monolayer (SAM) and the $\mathrm{AAO}$ has been overcome by modifying the surface chemistry of the fabricated AAO mold by coating it with thin $\mathrm{SiO}_{2}$ layer. Then we have applied the commonly used silane-based self-assembled monolayer (SAM) on these $\mathrm{SiO}_{2}$-coated AAO molds and achieved successful imprinting of resist pillars with feature size of $172 \pm 25 \mathrm{~nm}$ by using the mold with a pore diameter of $163 \pm 24 \mathrm{~nm}$. Finally, we have achieved (001) oriented $\mathrm{L}_{0}$ FePt patterned structure with a dot diameter of $42 \pm 4 \mathrm{~nm}$ by using a AO mold with a pore diameter of $73 \pm 7 \mathrm{~nm}$. The perpendicular $H_{c}$ of the unpatterned and patterned FePt is about $3.3 \mathrm{kOe}$ and $12 \mathrm{kOe}$, respectively. These results indicate that AAO mold can potentially be used in NIL for fabricating patterned nanostructures over large area.

\section{Introduction}

Nanoimprint lithography (NIL) has attracted enormous attentions [1-6] of the nanofabrication community since its innovation in 1995 [7] and regarded as a next generation nanofabrication tool for fabricating sub-10 nm features [35]. The NIL is a high-throughput and low-cost lithographic technique that showed its potential in many emerging fields, such as electronics [8], photonics [9], bit-patterned media $[10,11]$, and biological applications [12]. Bit patterned media is of particular interest due its potential in increasing the areal densities in magnetic data storage devices [13, 14]. The mold (also known as template or stamp) is the crucial part of the NIL that determines the throughput and resolution of the later in practical applications. Usually electron beam lithography or focused ion beam lithography followed by the pattern transfer using reactive ion etching (RIE) are used for making the mold. The high cost and low throughput of these methods limit the usefulness of the NIL for fabricating nanopatterns over large area for both of the research and practical applications. Therefore, exploring the fabrication of low-cost and highly robust large-area mold is a must to enjoy the full potential of the NIL. However, to date, only very few works have been reported in this highly demanding area. Park et al. [15] fabricated the large-area mold by using block-copolymer lithography that involved an extra-pattern transfer step. Kim et al. [2] and Lee et al. [16] used AAO membrane filled with carbon and aluminum, respectively, that also involved number of harsh steps, like fabrication of AAO membrane that is difficult to handle due to its brittle nature, attaching that AAO membrane with another rigid substrate, deposition of carbon or aluminum, heat treatment, ion milling, and chemical etching.

In this paper, we have demonstrated the direct use of AAO fabricated on an Si wafer as the mold by modifying the surface chemistry of the AAO mold by coating it with a thin $\mathrm{SiO}_{2}$ layer using atomic layer deposition (ALD). We have successfully fabricated $\mathrm{L}_{0}$ FePt nanostructure with $42 \pm 4 \mathrm{~nm}$ 
feature size over $1 \mathrm{~cm}^{2}$ area. Our developed mold is highly economic, and both of the lateral and vertical dimensions can be controlled easily $[17,18]$.

\section{Experimental Procedure}

The fabrication procedure is schematically shown in Figures $1(\mathrm{a})-1(\mathrm{~h})$. The procedure consists of two main processes: (i) preparation of the AAO mold with different pore diameter, coating it with a thin $\mathrm{SiO}_{2}$, followed by SAM deposition and (ii) nanoimprinting.

\subsection{Preparation of the AAO Mold with Different Pore Diam-} eter. At first, a $1000 \mathrm{~nm} \mathrm{Al}$ film with a thin $\mathrm{Ti}$ underlayer was deposited on a 4 inch $(\sim 10.2 \mathrm{~cm})$ circular disc of a $\mathrm{Si}$ wafer by thermal evaporation (Figure $1(\mathrm{a})$ ). This disc coated with $\mathrm{Al}$ film was cut into $30 \mathrm{~mm} \times 20 \mathrm{~mm}$ pieces and anodized in phosphoric and oxalic acid by a two-step anodization process as described elsewhere [18-21]. The first anodization was conducted under $40 \mathrm{~V}$ and $160 \mathrm{~V}$ in the $2.7 \mathrm{wt} \%$ oxalic acid $\left(\mathrm{H}_{2} \mathrm{C}_{2} \mathrm{O}_{4}\right)$ and $10 \mathrm{wt} \%$ phosphoric acid $\left(\mathrm{H}_{3} \mathrm{PO}_{4}\right)$, respectively, for specific time. Subsequently, the sample was immersed into a mixture solution of $\mathrm{H}_{3} \mathrm{PO}_{4}$ $\left(50 \mathrm{mLl}^{-1}\right)$ and $\mathrm{Cr}_{2} \mathrm{O}_{3}\left(30 \mathrm{gl}^{-1}\right)$ at $70^{\circ} \mathrm{C}$ for specific time to remove the alumina membrane formed during the first anodization, in order to obtain a highly ordered patterned $\mathrm{Al}$ surface. Then the second anodization was carried out with the condition the same as the first step. The anodization carried out in oxalic and phosphoric acid provided the small and large interpore distance, respectively. Then the pore widening treatment was conducted in $5 \mathrm{wt} \%$ phosphoric acid at $30^{\circ} \mathrm{C}$. The pore diameter of anodized alumina fabricated in phosphoric acid and oxalic acid after pore widening treatment is $163 \pm 24 \mathrm{~nm}$ and $73 \pm 7 \mathrm{~nm}$, respectively.

2.2. Nanoimprinting. The AAO molds were coated with a very thin $\sim 2-3 \mathrm{~nm} \mathrm{SiO}_{2}$ layer by atomic layer deposition (ALD) technique followed by the coating with a commonly used silane-based SAM (Heptadecafluoro-1,1,2,2-tetra-hydrodecyl trichlorosilane) (Figure 1(b)). The surface modification of the AAO by $\mathrm{SiO}_{2}$ was the key to the success of the direct use of AAO mold for NIL. The $\mathrm{L}_{0}$-ordered FePt $(4 \mathrm{~nm})$ continuous film with film structure of $\mathrm{Si} / \mathrm{SiO}_{2} /$ $\mathrm{CrRu}(30 \mathrm{~nm}) / \mathrm{Pt}(3 \mathrm{~nm}) / \mathrm{FePt}(4 \mathrm{~nm}) / \mathrm{Ru}(3 \mathrm{~nm})$ was grown by cosputtering from $\mathrm{Pt}$ and $\mathrm{Fe}$ targets at $350^{\circ} \mathrm{C}$ on $\mathrm{Si}$ wafer (Figure $1(\mathrm{c})$ ). Then the FePt film was coated with Nanonex-1050 thermal resist by spin coating (Figure 1(d)). Then thermal nanoimprinting was performed by using Nanonex BX200 nanoimprinter on Si wafer and FePt thin film spin coated with thermal resist (Figure 1(e)). The resist flown into the AAO pores remained as pillars after demolding (Figure 1(f)). The FePt film was patterned by RIE and Ar ion milling using the resist pillars as an etching mask (Figure $1(\mathrm{~g})$ ). The remaining resist pillars were removed by chemical etching in acetone and RIE that leaves FePt pillars on the substrate (Figure 1(h)).
The surface morphology of the mold and imprinted pattern structure was observed with a field-emission scanning electron microscope (FE-SEM). The magnetic properties of the FePt film before and after patterning were measured by a vibrating sample magnetometer (VSM) and a superconducting quantum interference device (SQUID), respectively. The crystallographic property of the continuous FePt film was analyzed by an X-ray diffractometer (XRD).

\section{Results and Discussion}

We have started with a mold with large pore diameter. Figure 2(a) shows SEM plane view image of the AAO mold fabricated in phosphoric acid. The inset shows the distribution of the AAO pore diameter. The diameter of the AAO pore is $163 \pm 24 \mathrm{~nm}$. The depth of the pore is about $400 \mathrm{~nm}$ estimated from an SEM cross-sectional image. Improvement of the ordering and size distribution of the $\mathrm{AAO}$ pores is beyond the scope of this work that can be achieved by optimizing the anodization parameters $[20,21]$. Figure 2(b) shows the SEM image of the imprinted resist on an Si wafer by using the mold shown in Figure 2(a) coated with the SAM layer only without any $\mathrm{SiO}_{2}$ coating. The imprinting is carried out at $350 \mathrm{psi}, 175^{\circ} \mathrm{C}$, for 3 minutes. The resist over more than $50 \%$ area is peeled off with the mold as represented by the bright areas. Although resist remains adhered on the rest part of the substrate, no features are observed as indicated by the high-magnification image of the corresponding area shown in the inset. The friction and sticking problem between the mold and the resist is one of the main challenges in nanoimprinting technology. With the nanostructure size becoming smaller and smaller, the surface-to-volume ratio increases dramatically and the friction and sticking problem becomes more severe. When the mold makes contact with the substrate coated with resist, the high surface energy produces a large amount of surface force. With the interaction between surfaces, the profiles of the two surfaces change and reestablish a new energy equilibrium state that produces sticking. Due to the sticking forces between surfaces, the nanosystem produces a large friction force, especially during the demolding process. Among the several options [22, 23] application of the silane-based hydrophobic self-assembled monolayer (SAM), namely, "Heptadecafluoro-1,1,2,2-tetrahydrodecyl Trichlorosilane," has been reported as the most successful lubrication method [23]. We do not get any imprinting by using this silane-based SAM directly on our AAO mold as shown in Figure 2(b) due to the inferior bonding between $\mathrm{Al}_{2} \mathrm{O}_{3}$ and the SAM layer. Then we modify the surface of the AAO mold by coating it with a very thin $\sim 2-3 \mathrm{~nm} \mathrm{SiO}_{2}$ by ALD followed by the deposition of the SAM layer on it. Eventually, we achieve excellent imprinting over more than $1 \mathrm{~cm}^{2}$ area with very good replication of the mold as shown in Figure 2(c). The imprinting from even the smallest features is also clearly shown in the inset of Figure 2(c). The diameter of the imprinted features is $172 \pm 25 \mathrm{~nm}$ as shown in the left inset of Figure 2(c). The diameter of imprinted resist pillars is slightly larger than the mold diameter may be due to the swelling of the resist by electron beam during SEM imaging. 


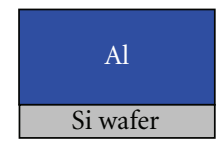

(a)

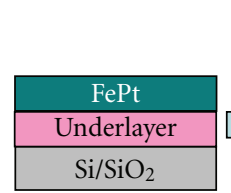

(c)

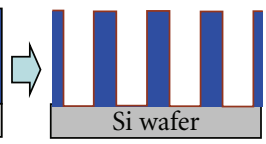

(b)

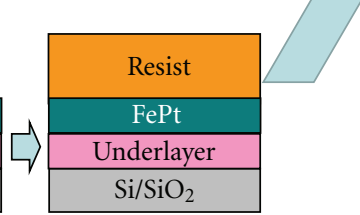

(d)

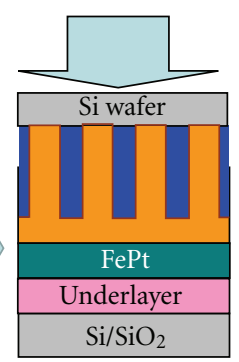

(e)

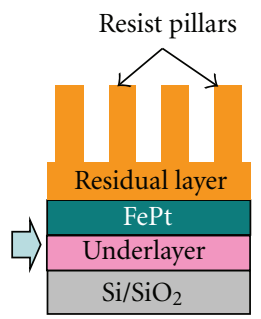

(f)

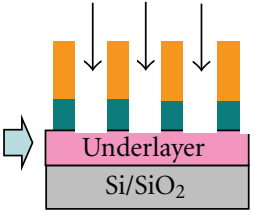

(g)

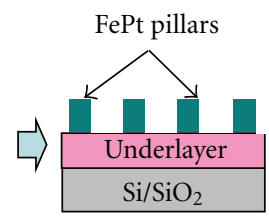

(h)

Figure 1: Schematic drawing of the fabrication of FePt patterned structure by using anodized alumina (AAO) mold. (a) Deposition of Al film on a Si wafer, (b) fabrication of AAO and coating it with thin $\mathrm{SiO}_{2}$ and SAM layer, (c) fabrication of $\mathrm{L}_{0}$ ordered FePt thin film, (d) spin coating of the resist on FePt thin film, (e) nanoimprinting, (f) demolding, (g) removal of the residual layer by reactive ion etching (RIE) and transfer of the pattern into FePt film by Ar ion milling, and (h) removal of the resist to leave the FePt pillars on the substrate.

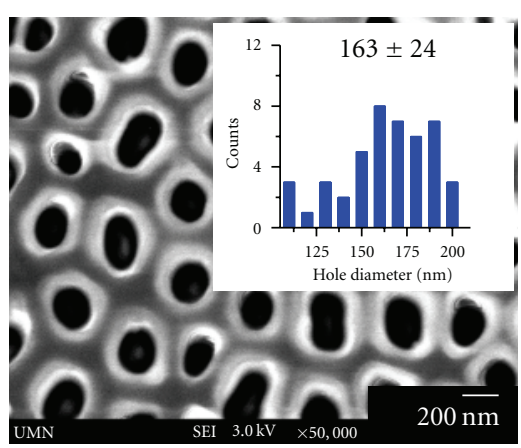

(a)

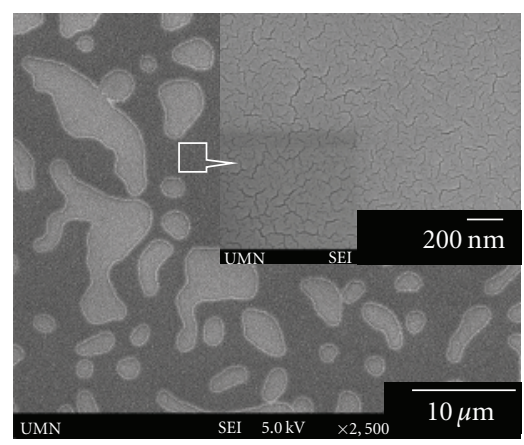

(b)

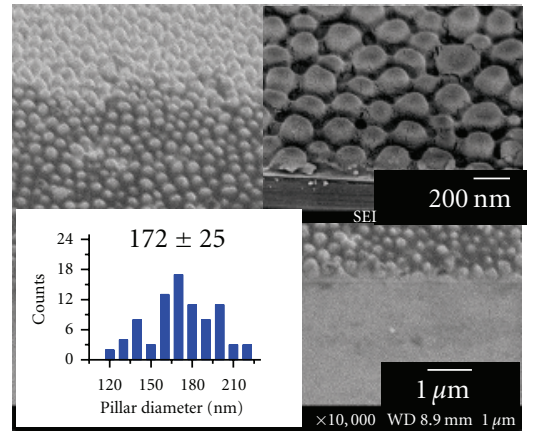

(c)

FIGURE 2: SEM plane-view images of (a) AAO mold; inset is the pore diameter distribution, (b) as-imprinted resist by using mold without $\mathrm{SiO}_{2}$ coating; inset is the magnified view of the indicated area, and (c) as-imprinted resist pillars by using the mold with thin $\sim 2-3 \mathrm{~nm} \mathrm{SiO}{ }_{2}$ coating. Insets in the upper right and left bottom corners are the magnified view and distribution of resist pillar diameter, respectively.

The coating of the AAO mold with $\mathrm{SiO}_{2}$ layer reduces the surface energy of the mold and enhances the bonding with SAM layer, which is the key of getting successful imprinting.

After achieving successful imprinting of resist pillars with large feature size on a $\mathrm{Si}$ wafer, we concentrate our efforts on reducing the feature size and fabricate $\mathrm{L} 1_{0} \mathrm{FePt}$ patterned structure. We grow $\mathrm{L}_{0}$-ordered $\mathrm{FePt}(4 \mathrm{~nm})$ continuous film with film structure of $\mathrm{Si} / \mathrm{SiO}_{2} / \mathrm{CrRu}(30 \mathrm{~nm}) /$ $\mathrm{Pt}(3 \mathrm{~nm}) / \mathrm{FePt}(4 \mathrm{~nm}) / \mathrm{Ru}(3 \mathrm{~nm})$. The FePt layer is cosputtered from Pt and $\mathrm{Fe}$ targets at $350^{\circ} \mathrm{C}$. This FePt thin film is spin-coated with $150 \mathrm{~nm}$ thick thermal resist and patterned by using an AAO mold with $73 \pm 7 \mathrm{~nm}$ pore diameter.

Figure 3(a) shows the plane-view SEM image of the mold. This mold is fabricated by anodizing the $\mathrm{Al}$ film in oxalic acid to get smaller pore diameter [21]. The area of the mold is about $1 \mathrm{~cm}^{2}$. The diameter of the pore in the mold is $73 \pm 7 \mathrm{~nm}$ as shown in the inset of Figure 3(a), and the depth of the pore is about $150 \mathrm{~nm}$ estimated from an SEM cross-sectional image. The imprinting is performed at $350 \mathrm{psi}, 175^{\circ} \mathrm{C}$, for 3 minutes. Figure $3(\mathrm{~b})$ shows the SEM cross-sectional image of as imprinted resist pillars on $\mathrm{FePt}$ thin film and the inset shows the distribution of resist pillar diameter. The diameter of the imprinted resist pillar is $76 \pm$ $9 \mathrm{~nm}$. The height of some of the pillars is smaller than that of the others as indicated by the white arrows. This may be due to the lack of sufficient flatness of the mold and insufficient initial resist thickness. Figure 3(c) shows the plane-view SEM image of the FePt dots on the Si wafer after removing the residual layer and transferring the pattern by Ar ion milling using the resist pillars as an etching mask. The diameter of the FePt dots is $42 \pm 4 \mathrm{~nm}$. The FePt dot diameter is about $45 \%$ smaller than the pore diameter of the mold as well as the as-imprinted resist pillars. This size reduction may occur due to the lateral etching of the resist pillars during residual layer removal by RIE. This size reduction also indicates that the size of the imprinted features can again be tuned by controlling the pore depth and residual layer thickness. Some of the dots as indicated by the black arrows in the inset of Figure 3(c) are almost etched away due to the smaller mask height onto it as indicated by the white arrows in Figure 3(b). As a result, the density of the FePt dots is somewhat smaller than the density of the pores in the mold. 


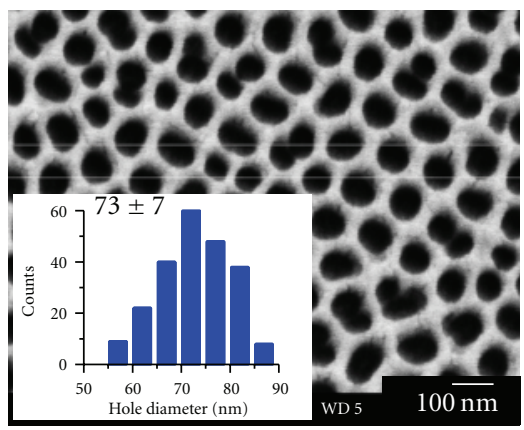

(a)

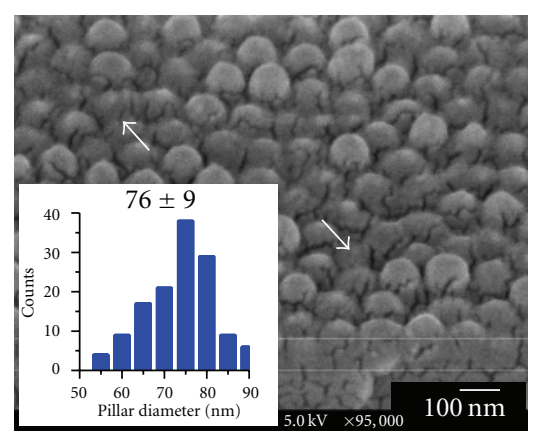

(b)

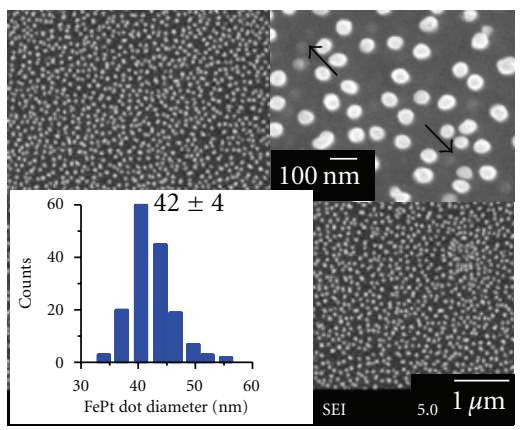

(c)

FIGURE 3: SEM plane-view images of (a) AAO mold coated with thin $\mathrm{SiO}_{2}$ layer; inset is the distribution of pore diameter of the mold, (b) the cross-sectional SEM image of as-imprinted resist pillars, and inset is the distribution of resist pillar diameter. Some pillars with smaller height are indicated by white arrows, and (c) plane-view SEM image of FePt dots on Si wafer; inset in the upper right corner is the magnified view and in left bottom corner is the size distribution of FePt dots. Some dots with reduced height are indicated by black arrows in the magnified view.

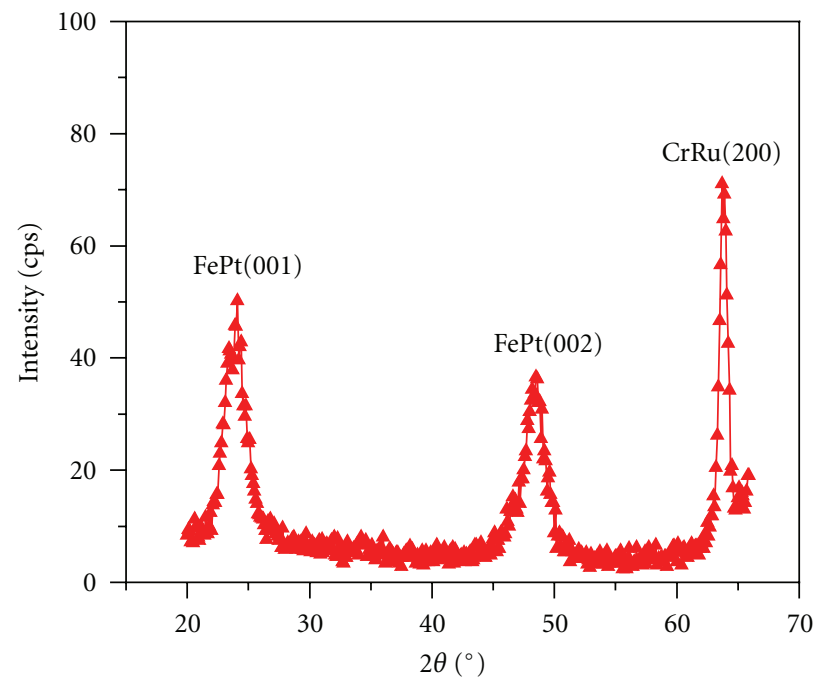

(a)

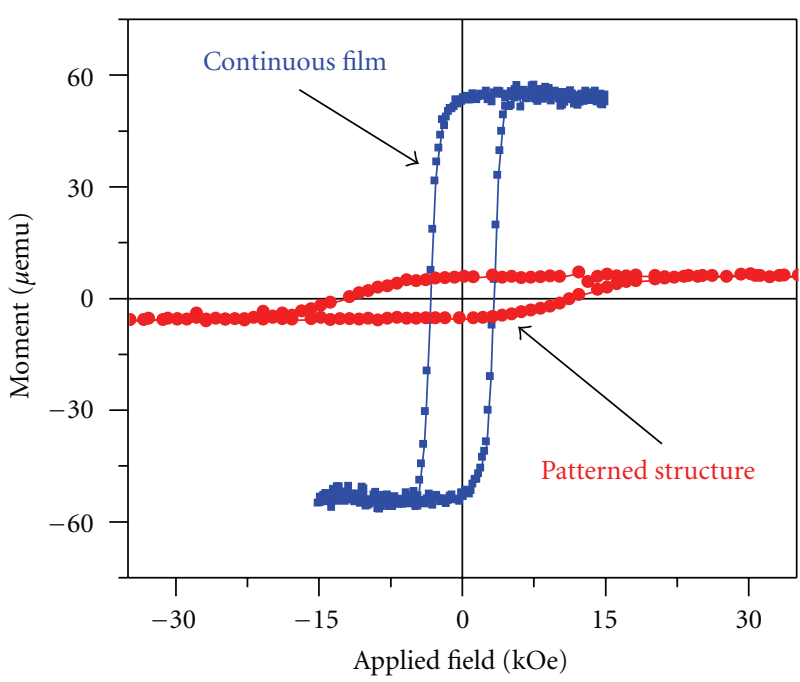

(b)

Figure 4: (a) XRD diagram of continuous FePt film and (b) $M-H$ loops of continuous film and patterned FePt structure as marked. The size for the magnetic measurement of both samples was $0.25 \mathrm{~cm}^{2}$.

Figures 4(a) and 4(b) show the XRD pattern of the FePt continuous film and $M-H$ loops of the continuous film and patterned structure, respectively. The XRD pattern of the continuous FePt film before patterning exhibits very good $\mathrm{L} 1_{0} \mathrm{FePt}$ (001) texture. The FePt continuous film exhibit very good perpendicular anisotropy with out-of-plane $H_{c}$ of $3.3 \mathrm{kOe}$ and a squareness ratio of about unity that is good in agreement with the good (001) texture in the XRD diagram. The magnetic property of the patterned FePt structure is measured by using a SQUID at room temperature. The perpendicular coercivity of the patterned structure is about $12 \mathrm{kOe}$, that is, about 3.5 times larger than that of the continuous film. This $H_{c}$ value is much lower than that of the isolated FePt islands on $\mathrm{MgO}$ [24]. Several mechanisms can be considered for this low $H_{c}$ value of the patterned structure. First, diffusion may occur at the interface between the $\mathrm{Pt}$ layer and the FePt layer as $\mathrm{Pt}$ is used as interlayer; therefore, the composition and switching field at the interface may differ from the other part. The FePt at the interface with smaller switching field may be exchange coupled with the adjacent part with larger switching field and reduces the $H_{c}[17,25]$. Second, the redeposition effect may add some disordered FePt onto the outer surface of the pillars. This re-deposited disordered FePt may act as nucleation sites to start the magnetization switching at low field that can reduce the coercivity [26]. In addition, the edge damage of the FePt pillars by ion milling may also be one of the reasons for the low $H_{c}$ in patterned FePt structure [27].

\section{Conclusions}

We have shown that the anodized alumina (AAO) fabricated on Si wafer can be used as a large area mold for nanoimprinting. The sticking problem between the mold and the 
resist has been overcome by modifying the AAO surface by coating it with a very thin $\mathrm{SiO}_{2}$ layer. Moreover, we have successfully fabricated $\mathrm{L}_{0} \mathrm{FePt}$ patterned structure with $42 \pm 4 \mathrm{~nm}$ feature size. Optimization of nanoimprinting parameters and flatness of the mold may open the path to fabricate patterned magnetic nanostructures with reduced feature size and high density for practical applications.

\section{Acknowledgments}

This work is partially supported by the INSIC's extremely high areal density recording (EHDR) program and the National Science Foundation (NSF) MRSEC program. A part of the of the work has been carried out in the Nano Fabrication Center (NFC) and Characterization Facilities, the University of Minnesota. The authors are thankful to Dr. Mark Fisher at the NFC, University of Minnesota.

\section{References}

[1] D. R. Barbero, M. S. M. Saifullah, P. Hoffmann et al., "High resolution nanoimprinting with a robust and reusable polymer mold," Advanced Functional Materials, vol. 17, no. 14, pp. 2419-2425, 2007.

[2] Y. S. Kim, K. Lee, J. S. Lee, G. Y. Jung, and W. B. Kim, "Nanoimprint lithography patterns with a vertically aligned nanoscale tubular carbon structure," Nanotechnology, vol. 19, no. 36, Article ID 365305, 2008.

[3] Z. Li, Y. Gu, L. Wangs et al., "Hybrid nanoimprint-soft lithography with sub-15 nm resolution," Nano Letters, vol. 9, no. 6, pp. 2306-2310, 2009.

[4] M. Schvartzman and S. J. Wind, "Robust pattern transfer of nanoimprinted features for sub-5-nm fabrication," Nano Letters, vol. 9, no. 10, pp. 3629-3634, 2009.

[5] J. Haisma, M. Verheijen, K. Van Den Heuvel, and J. Van Den Berg, "Mold-assisted nanolithography: a process for reliable pattern replication," Journal of Vacuum Science and Technology $B$, vol. 14, no. 6, pp. 4124-4128, 1996.

[6] P. F. Murphy, K. J. Morton, Z. Fu, and S. Y. Chou, "Nanoimprint mold fabrication and replication by room-temperature conformal chemical vapor deposition," Applied Physics Letters, vol. 90, no. 20, Article ID 203115, 2007.

[7] S. Y. Chou, P. R. Krauss, and P. J. Renstrom, "Imprint of sub$25 \mathrm{~nm}$ vias and trenches in polymers," Applied Physics Letters, vol. 67, p. 3114, 1995.

[8] E. Menard, M. A. Meitl, Y. Sun et al., "Micro- and nanopatterning techniques for organic electronic and optoelectronic systems," Chemical Reviews, vol. 107, no. 4, pp. 1117-1160, 2007.

[9] L. J. Guo, "Recent progress in nanoimprint technology and its applications," Journal of Physics D, vol. 37, no. 11, pp. R123R141, 2004.

[10] P. R. Krauss and S. Y. Chou, "Nano-compact disks with 400 Gbit/in2 storage density fabricated using nanoimprint lithography and read with proximal probe," Applied Physics Letters, vol. 71, no. 21, pp. 3174-3176, 1997.

[11] J. S. Sohn, D. Lee, E. Cho et al., "The fabrication of CoPt electro-deposited bit patterned media with nanoimprint lithography," Nanotechnology, vol. 20, no. 2, Article ID 025302, 2009.
[12] V. N. Truskett and M. P. C. Watts, "Trends in imprint lithography for biological applications," Trends in Biotechnology, vol. 24, no. 7, pp. 312-317, 2006.

[13] C. A. Ross, "Patterned magnetic recording media," Annual Review of Materials Science, vol. 31, pp. 203-235, 2001.

[14] B. D. Terris and T. Thomson, "Nanofabricated and selfassembled magnetic structures as data storage media," Journal of Physics D, vol. 38, no. 12, pp. R199-R222, 2005.

[15] H. J. Park, M. G. Kang, and L. J. Guo, "Large area high density sub-20 $\mathrm{nm} \mathrm{SiO}_{2}$ nanostructures fabricated by block copolymer template for nanoimprint lithography," ACS Nano, vol. 3, no. 9, pp. 2601-2608, 2009.

[16] P. S. Lee, O. J. Lee, S. K. Hwang, S. H. Jung, S. E. Jee, and K. H. Lee, "Vertically aligned nanopillar arrays with hard skins using anodic aluminum oxide for nano imprint lithography," Chemistry of Materials, vol. 17, no. 24, pp. 6181-6185, 2005.

[17] M. T. Rahman, R. K. Dumas, N. Eibagi et al., "Controlling magnetization reversal in $\mathrm{Co} / \mathrm{Pt}$ nanostructures with perpendicular anisotropy," Applied Physics Letters, vol. 94, no. 4, Article ID 042507, 2009.

[18] M. T. Rahman, N. N. Shams, C. H. Lai, J. Fidler, and D. Suess, "Co/Pt perpendicular antidot arrays with engineered feature size and magnetic properties fabricated on anodic aluminum oxide templates," Physical Review B, vol. 81, no. 1, Article ID 014418, 2010.

[19] M. T. Rahman, N. N. Shams, and C. H. Lai, "A large-area mesoporous array of magnetic nanostructure with perpendicular anisotropy integrated on Si wafers," Nanotechnology, vol. 19, no. 32, Article ID 325302, 2008.

[20] H. Masuda, K. Yada, and A. Osaka, "Self-ordering of cell configuration of anodic porous alumina with large-size pores in phosphoric acid solution," Japanese Journal of Applied Physics, vol. 37, no. 11, pp. L1340-L1342, 1998.

[21] A. P. Li, F. Müller, A. Bimer, K. Nielsch, and U. Gösele, "Hexagonal pore arrays with a 50-420 nm interpore distance formed by self-organization in anodic alumina," Journal of Applied Physics, vol. 84, no. 11, pp. 6023-6026, 1998.

[22] K. I. Nakamatsu, N. Yamada, K. Kanda, Y. Haruyama, and S. Matsui, "Fluorinated diamond-like carbon coating as antisticking layer on nanoimprint mold," Japanese Journal of Applied Physics, vol. 45, no. 33-36, pp. L954-L956, 2006.

[23] K. J. Byeon, K. Y. Yang, and H. Lee, "Thermal imprint lithography using sub-micron sized nickel template coated with thin SiO2 layer," Microelectronic Engineering, vol. 84, no. 5-8, pp. 1003-1006, 2007.

[24] B. Ma, H. Wang, H. Zhao, C. Sun, R. Acharya, and J. P. Wang, "L10 FePt/Fe exchange coupled composite structure on $\mathrm{MgO}$ substrates," IEEE Transactions on Magnetics, vol. 46, no. 6, Article ID 5467459, pp. 2345-2348, 2010.

[25] J. P. Wang, W. Shen, and S. Y. Hong, "Fabrication and characterization of exchange coupled composite media," IEEE Transactions on Magnetics, vol. 43, no. 2, pp. 682-686, 2007.

[26] T. Seki, T. Shima, K. Yakushiji, K. Takanashi, G. Q. Li, and S. Ishio, "Improvement of hard magnetic properties in microfabricated L1 0 -FePt dot arrays upon post-annealing," IEEE Transactions on Magnetics, vol. 41, no. 10, pp. 3604-3606, 2005.

[27] J. M. Shaw, S. E. Russek, T. Thomson et al., "Reversal mechanisms in perpendicularly magnetized nanostructures," Physical Review B, vol. 78, no. 2, Article ID 024414, 2008. 

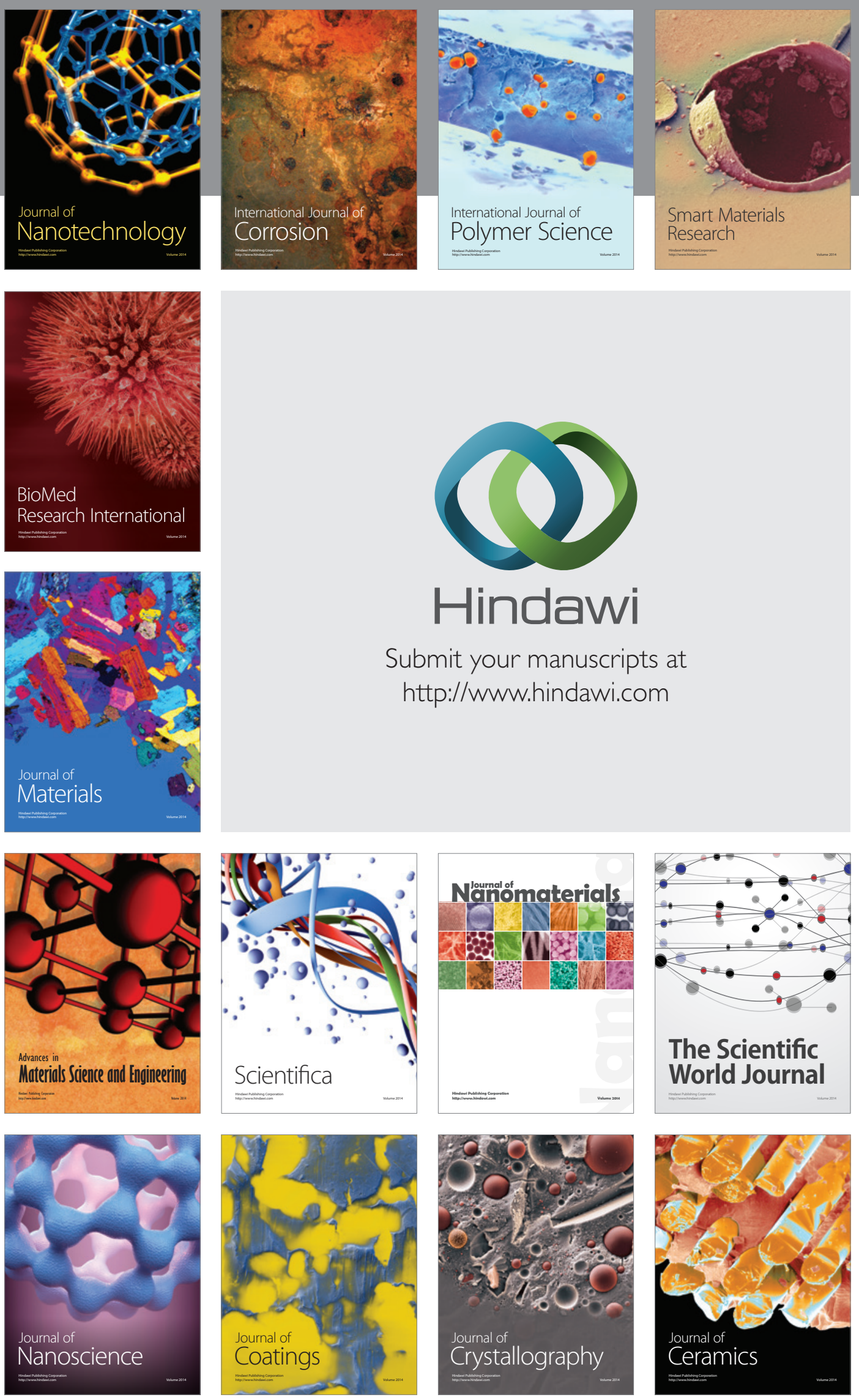

The Scientific World Journal

Submit your manuscripts at

http://www.hindawi.com

\section{World Journal}

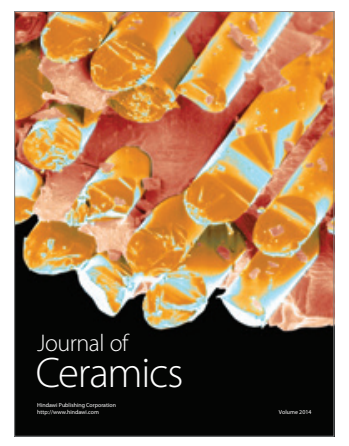

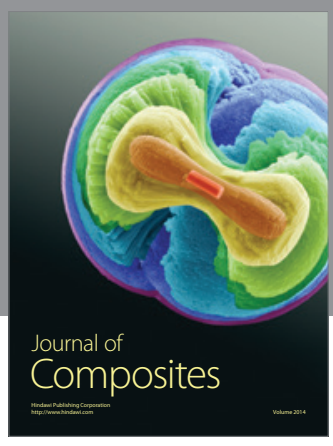
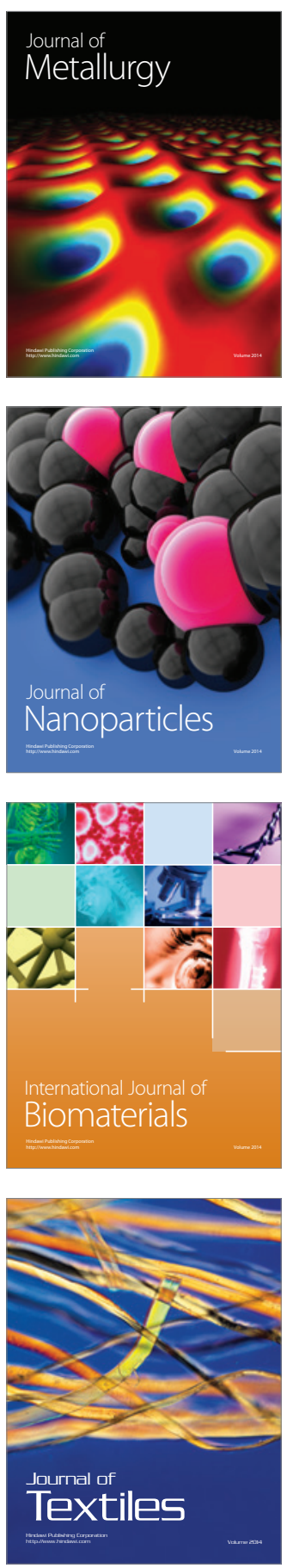